\title{
Distributed Maintenance of Multiple Product Views
}

\author{
Christoph M. Hoffmann * \\ Department of Computer Sciences \\ Purdue University \\ Robert Joan-Arinyo ${ }^{\dagger}$ \\ Departament de Llenguatges i Sistemes Informàtics \\ Universitat Politècnica de Catalunya
}

September 18, 1998

\begin{abstract}
We present three mechanisms for maintaining consistent product views in a distributed product information data base. The mechanisms are used when one of the views makes a change to the product model and the other views must be updated to maintain consistency.
\end{abstract}

Keywords: CAD, Master Model, Distributed Data, Product View, Features, Constraints, Neutral Representations.

\section{Introduction}

Data bases are an important element in discrete product design and manufacture. A key component is the CAD model that primarily captures the shape design, but increasingly has been enriched with other design and analysis data. The integration of different product information domains has evolved into the concept of a master model, a single repository in which resides all relevant product data. The master model concept has been embraced by industry, but it raises significant technical problems that continue to be studied in research.

Different activities in product design and manufacture examine different subsets of the information in the master model. The presentation of such an information subset has been called a view, [4]. Maintaining views consistently

\footnotetext{
${ }^{*}$ Supported in part by ONR Contract N00014-96-1-0635 and by NSF Grants CDA 92-23502 and CCR 95-05745.

${ }^{\dagger}$ While on leave in the Department of Computer Sciences, Purdue University. Partially supported by the CICYT Spanish Research Agency under Grant TIC95-0630-C05-04.
} 
is a central problem in research on product design and manufacture and is the subject of this paper.

In [16] we have proposed a distributed approach to the creation and maintenance of a master model data base. We favor the distributed approach for two reasons:

1. The creation of a monolithic system from the ground up is, in our opinion, not only difficult, but also leads to a realization that would pose difficult software maintenance problems. Creating a master model repository by combining collaborating, but otherwise autonomous, subsystems offers more flexibility and a greater ability to adapt to new information domains that should be integrated in the future.

2. It appears unrealistic to expect that industry would rely on a single system and a single software provider. The typical situation is that a company is committed to a specific CAD vendor whose CAD data is partially inaccessible except through the CAD system, and that this partially opaque data has to be integrated with other data, possibly of corporate origin and often inaccessible to the $\mathrm{CAD}$ vendor for proprietary reasons.

So, the distributed federation approach to fashioning a master model repository appears to be a practical one. It also poses some interesting intellectual challenges.

In the earlier paper, we identified two mechanisms for maintaining consistency of view, an external information association mechanism, and a constraint reconciliation procedure. We expand on the details and applicability of those mechanisms and add a third mechanism as a complementary technique for maintaining consistent views under distributed updates. We distinguish several categories of master model update:

1. shape changes,

2. changes of parameters, dimensions, and constraints, and

3. changes of attributes.

Of these, shape changes are the most difficult ones to respond to, and we cannot claim that all changes can be accommodated automatically. Therefore, restrictions on the shape changes will be imposed which, when exceeded, human intervention is required - absent new technological break throughs. The imposed restrictions will be discussed in detail and justified.

\section{Prior Work}

Recently, efforts have been made to respond to the increasing need for tools to concurrently conduct design, manufacturing and related activities in the product 
life cycle. It is widely accepted that these activities should be carried out in distributed and heterogeneous computing environments sharing a unique product model to guarantee consistent information, [1,2].

Distributed and heterogeneous computing environments have been studied in several recent works. Han and Requicha in [15] report on the implementation of a distributed environment encompassing a simple feature-based design system, a geometric server, an automatic feature recognizer and a graphics renderer, all running as separate processes. The geometric server is a central server and the other components are the clients. The motivation of this work was to interface a unique feature recognizer with several solid modellers. The goal is achieved by building the geometric server as a set of solid modelers each augmented with a software wrapper called adaptor that provides a uniform application programming interface. In this environment the geometric server stores both the net shape generated by the design system and the features extracted by the feature recognizer.

De Martino et al. present in [11] a distributed, object-oriented, featurebased system. An intermediate modeller acts as a server for a number of networked, distributed application clients. The intermediate modeller maintains a homogeneus, multiple-view, feature-based representation of the part. From this model, specific views can be derived. To maintain consistency between different views, only the designer client is allowed to modify the model. In this system, a single data structure stores the information of all the views.

Hoffmann and Joan-Arinyo, [16], developed an architecture for a product master model that federates CAD systems with downstream application processes for different feature views that are part of the design process. The architecture, based on a server-client model, addresses especially the need to make persistent associations of design information with net shape elements. Moreover, the design respects the need of commercial CAD systems (and of downstream application clients) to maintain proprietary information that must not be disclosed in the master model.

To deal with the consistency and association problems systems are organized as either one-way or multi-way architectures. In one-way architectures, features in an application view are derived from the features that belong to a privileged view, usually the design view. The designer defines this view and conversion modules derive application-dependent feature models. If a modification is required by a downstream application, it must be entered in the privileged view first. Only thereafter new application-dependent views can be derived, $[7,11,15]$. In this one-way approach, feature conversion is triggered by one of two different strategies. In one strategy, feature conversion is delayed until the design is considered completed, [11]. In the other strategy, also called incremental feature recognition, the conversion process is triggered immediately after the completion of each feature attachment operation in the design view, $[13,18,20]$. 
In multi-way architectures, modifications required by an application are introduced in the view in which the need for them arises, and all modifications in any view should be propagated automatically to all other views, [4, 10, 16]. Work based on this assumption may neglect to explain precisely how a feature view, other than the design view, can change the net shape of the design, and there is a paucity of techniques to formalize such changes. The work by Bronsvoort et al. is a notable proposal in that respect, $[4,8,9,12]$. This work models the net shape by a cell complex where the cells are refined such that every feature of an application view is composed of entire cells. That permits to edit shape mechanically in any feature view and to achieve consistency across all views using constraint techniques. If an inconsistency between different views is found, the approach rebuilds the view that generated the inconsistency. The view is rebuilt incrementally by first removing some features and then adding new features.

In this paper, we continue to explore the architecture proposed by us in [16]. We examine first net shape changes that can be accommodated by other clients purely by constraint schema reconciliation ([16]). Next, we consider the effect of a net shape edit in which the feature structures of different client views need to be rebuilt. Here, we propose an algorithm that restructures the feature view of a client that did not edit the net shape, to make that structure consistent with the new net shape. Only if the algorithms fails do we require human intervention. Finally, we revisit the question of updating nongeometric information and relationships that are associated with the net shape elements.

\section{$3 \quad$ Master Model Server and Clients}

We assume the master model (MM) scenario described in [16]. There is an object-oriented MM server that records all information to be shared explicitly among the participating subsystems. The clients that connect to the MM server are assumed to be autonomous but collaborating. That is, they may be in possession of undisclosed proprietary information that is important to their role in product design and analysis, but they pledge to follow the protocols and conventions of the MM server and, in particluar, disclose what shared information in the MM server repository is of interest to them.

The MM server receives notifications from a client that wishes to edit the product model, according to its own view. The client then proposes the edit and transmits the changes to the MM server. The MM server processes the changes and notifies the other clients that are affected by those changes in accordance with the protocols explained in [16]. If every interested client can successfully update, then the MM repository commits to the change and the edit proposed by the client is successful. Otherwise, the edit must be rolled back. We omit a description of the routine mechanisms needed to implement distributed, object- 
oriented data bases and the various mechanisms for locking and committing to such transactions.

The CAD system is one of the clients of the MM server. It is assumed that the CAD client publishes at least the net shape for deposition in the MM server. Feature information may, but need not, be published, as well as dimensional and constraint information. Note that this information can be published explicitly as a neutral data structure, or implicitly as a set of interrogating methods that produce information in response to queries. In an object-oriented MM server this distinction can be made transparent, perhaps with differentials in performance.

Accounting for shape changes requires a persistent naming mechanism and a protocol for expressing change. Persistent naming is, in essence, a mapping from shape elements (vertices, edges and faces) of the old net shape to those of the edited net shape. It is supported to by most CAD systems, in the following way:

An application program accesses the shape generated by the CAD system and asks for a persistent name $J$ of a particular face. $J$ is generated by the CAD system. After the CAD system has made some edits, the new shape is likely a different data structure. Now the application program can ask the CAD system "tell me the face that has the persistent name $J$." The CAD program may respond with an identification of a face in the new net shape, or else that the face is no longer present ans that the persistent name has become invalid.

Note that this persistence mechanism can be used to associate attributes or relations with shape elements, such as surface finish or tolerances. Such external associations can be updated automatically after edits by the associating application without the explicit involvement of the CAD system, at least for many updates. It is therefore a basis for distributed maintenance of product information.

As explained in [16], more information is desirable to manage external associations. Specifically, the change protocol is a neutral, qualitative description of the shape change that allows a greater degree of automatic external re-association. Moreover, the change protocol does not disclose proprietary methods the CAD system might use internally to manage persistence, and is therefore a realistic candidate for practical use.

\section{Synopsis of the Results}

We assume a MM server as described before, and we are coordinating two separate views, by two clients. Each view has access to the net shape in the MM server, but may have a different feature decomposition of the net shape. Each view maintains features and constraints. In addition, there are certain attributes 
associated with elements of the net shape in each view. We consider three types of edits and how they must be made consistent in the views:

1. In one view, a dimensional constraint is changed, and with it, the net shape. Update the other view consistent with the new net shape.

2. In one view, a feature is added or deleted, deriving a new net shape. Update the feature structure of the other view consistent with the new net shape.

3. In one view, a shape change has been made that affects relationships among net shape elements in the other view. Update the relationships of the other view consistent with the change.

For the sake of specificity, we illustrate these edit operations by using a CAD client and a machining process planning (MPP) client. The relationships considered are geometric dimensioning and tolerancing (GD\&T) relationships.

We consider only changes in a product view that affect the net shape. Mechanical artifacts perform their function primarily through the interaction of shapes. Therefore, this assumption is not a strong restriction. Since geometric shape can be structured in many different ways, the main obstacle to coordinating views is that one view does not necessarily understand how another view structures the same shape. Therefore, it would be meaningless to announce to the MM server that feature $\mathrm{X}$ has been deleted from view $\mathrm{Y}$, or that a particular dimension has been changed.

\subsection{Change of a Dimension}

A feature may be modified by changing a dimensional constraint. If the change does not alter the topology, this type of change is a good candidate for constraint reconciliation proposed in [16]. When the topology does change, the modification should be treated as a feature change. The change of a geometric constraint, for instance dropping a perpendicularity requirement or changing it to a specific angle, is approached in the same way.

\subsection{Feature Change}

We restrict to features that are extrusions and revolutions only. We assume that each feature makes a direct contribution to some of the faces of the net shape. Situations illustrated in Figure 1 are not considered. When a feature is added, deleted, or modified, the new net shape is communicated to the MM server, and the change protocol details how the new net shape relates to the old net shape.

A different view updates by an algorithm that adjusts this view's feature structure to replicate the new net shape. The algorithm may backtrack and

could fail. However, we can know in principle every way in which the same 

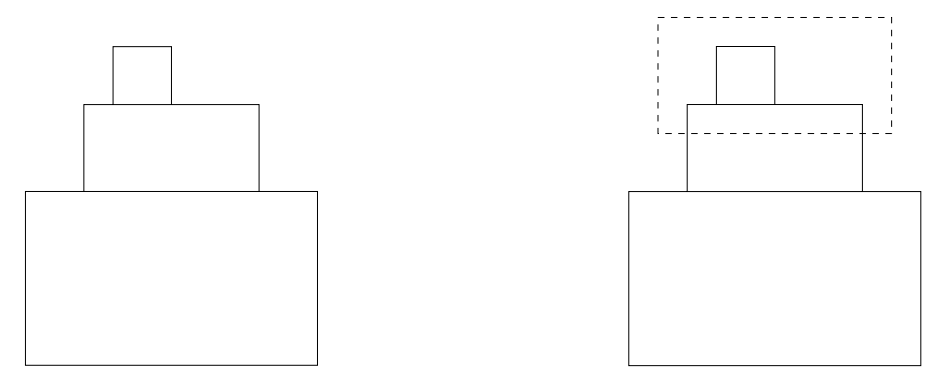

Figure 1: Left: The base feature $F_{0}$ is a block and is angmented by two extrusion features $F_{1}$ and $F_{2}$. Right: a cut of the dashed shape would eliminate $F_{2}$ altogether. Since $F_{2}$ does not contribute to the net shape, it ought to be dropped from the feature structure.

shape can be re-interpreted, and we consider reinterpretation as part of the algorithm. We do not attempt to develop feature re-ordering algorithms.

\subsection{Implied Attribute and Relationship Changes}

In some cases, the change of net shape requires a change, in a different view, of an attribute of a net shape element, or of a relationship among several net shape elements. Here, we propose a rule-based update procedure that accounts for the specific nature of the attribute or relationship. The rules are individual to the nature of the attribute, but the way in which they are evaluated is general. The algorithm, first sketched in [16], applies the rules by a propagation through the change protocol.

\section{$5 \quad$ Feature Views and Changes}

We assume that the detailed feature structure maintained by a view is not disclosed to the MM server and remains private information of each client. We want to maintain consistent but different views.

Client 1 updates the net shape with some feature editing operation and derives a new net shape. We consider how client 2 can update its own feature structure and obtain a consistent reinterpretation of the new net shape. In general, this is a rather broad problem statement, and a fully automated solution would include many techniques from feature recognition. See, for example, $[3,14,20,21,22]$ and references therein. We restrict the problem and the geometric operations, and allow the reinterpretation process to fail. If it fails, human intervention is required. Moreover, some adjustments, when performed automatically, should be reported to the user if they might violate design decisions the other view has been unaware of. Such notification is important in practice, but is not discussed here. 


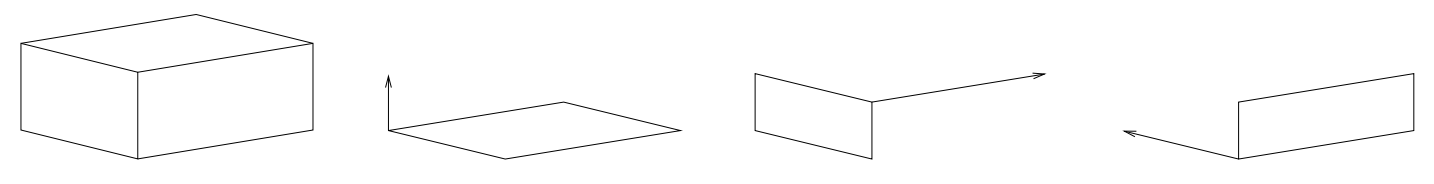

Figure 2: Three ways to create a parallelepiped

Recall that we assume that the features are cuts and protrusions that subtract from or add to the netshape. The generation method is assumed to be by extrusion or revolution. We exclude operations such as shelling, drafting, and blending. Note that the latter two could be derived by detailing steps from an appropriate attributation of the shape design. Finally, we consider the following attachment operations: from - to with explicitly designated targets, from-prev, through-next, from all, through all, and blind with a numerical extent; see also [6].

\subsection{Feature Reinterpretation}

We will allow feature update operations that redefine the feature generation method. Recall from [6] the notion of a proto feature. In the case of an extruded feature, the proto feature is a blind extrusion, to a particular extent, of the cross section, so that the chosen attachment attributes can be conceptualized as Boolean operations on the proto feature and the prior geometric shape. In the case of a revolved feature, the proto feature is a blind revolution, again of sufficient extent.

An elementary reinterpretation is a change in the generation method and cross section of a proto feature such that the same surfaces are generated but in

a different way. The following cases are considered elementary reinterpretations:

Parallelepiped extrusion: The proto feature is shown in Figure 2 and can be obtained from three different cross sections by extrusions in three directions. Note that the extrusion directions may be at an angle to the cross section plane.

Cylindrical revolution: The proto feature is shown in Figure 3 and can be obtained by a revolution or by an extrusion.

When necessary, we allow switching from one interpretation to another one.

For example, assume that the cylinder of Figure 3 was viewed by client 1 as a revolution and by client 2 as an extrusion, then a change in the cross section by client 1 may destroy the interpretation of client 2 , and we may have to change the feature definition of client 2 from an extrusion to a revolution.

A general reinterpretation is the reinterpretation of a feature as a group of features, by a decomposition. For example, assume that client 1 creates a cylinder feature by revolution that has been interpreted as an extrusion by client 

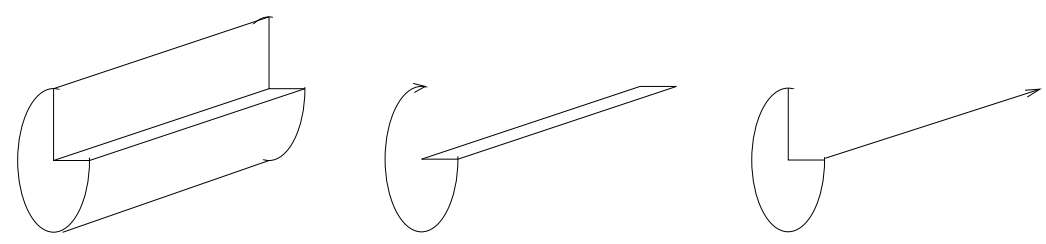

Figure 3: Two ways to create a cylinder

2. Client 1 edits the cross section, obtaining the new feature shown in Figure 4. The modification invalidates the extrusion interpretation of client 2. However, by splitting the extrusion into two separate extrusions, client 2 can maintain the interpretation.

Now consider any net shape obtained by the extrusion and revolution operations coupled with the attachment rules from before. Every surface is either a cylindrical or a revolute surface. Planar surfaces and surfaces on a right-circular cylinder are considered a special case of both the cylindrical and revolute surface type. Every cylindrical surfaces can be obtained by an extrusion, and every revolute surface by a revolution, from a suitable cross section element. Moreover, surfaces that are both revolute and cylindrical may allow elementary reinterpretation, depending on how they are delimited.

When generating a feature, additional surfaces may be obtained by the attachment rules that are not part of the extrusion or the revolution. Therefore, except for such surfaces, different feature structures of the same net shape arise only as follows:

1. a parallel extrusion has an overlapping cross section;

2. a concentric revolution has an overlapping cross section;

3. an elementary reinterpretation of a part of the feature.

Note that the attachment rules blur recognition of these cases because, by feature collision, they may create delimiting feature surfaces that appear to exclude elementary reinterpretation. By considering first the proto feature shape this difficulty is ameliorated.

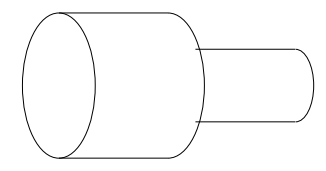

Figure 4: One revolved feature or two extruded features 


\section{Change of a Dimension}

When the editing client alters only constraints, under the assumption of topological invariance, the net shape change is such that the number of features and their interdependence in the updating client remains the same. Updating client structures can be made consitent with the new net shape either by reconciliation or by history-based adjustment.

\subsection{Adjustment by Reconciliation}

When the editing client alters only constraints, there is the possibility that the change impacts a single cross section only. Since the net shape mapping is communicated, it is possible to notify the MM server that the new net shape came about by constraint changes and what the geometric elements are on which the constraint is defined. If those elements are generated by a single cross section and the prior geometry, in the updating client, constraint reconciliation can be used as described in [16].

At this time, the majority of CAD designs are history-driven, and the major CAD systems do not use variational spatial constraints to define a shape design. Because of this limitation, constraint reconciliation is restricted to those constraints that map, in the editing client, to a single feature. For other constraint changes we advocate the techniques explained later on.

Since the change protocol has identified all net shape alterations, including face relocations, we can identify the cross section impacted by a constraint change and recognize the fact that reconciliation is applicable. In the following we assume that a single constraint has changed. The case of multiple changes is a straightforward extension.

In general, the changed constraint does not appear explicitly in the affected cross section. It is possible, in principle, to express the functional dependence of the changed constraint on the other constraints in the cross section, and to derive from that dependence how the constraint values of the updating client should be changed. However, those dependencies can become intractable symbolic expressions, and it is much simpler to take a procedural approach.

Since the new constraint has been used to derive the new net shape, a cross section recomputation, from the new net shape, yields the appropriate cross section. From this cross section, the constraint values for the updating client can be computed by measurement. Thereafter, we must verify that the new cross section correctly generates the new net shape.

The recomputation of the cross section involves both geometric changes to the cross section and changes to the constraint schema. Constraint changes may be trivial, such as the change of an angle or distance constraint, or they may be exceptional, such as the change of a parallel constraint to a nonzero angle, the change of an incidence constraint to a nonzero distance, and so on. 

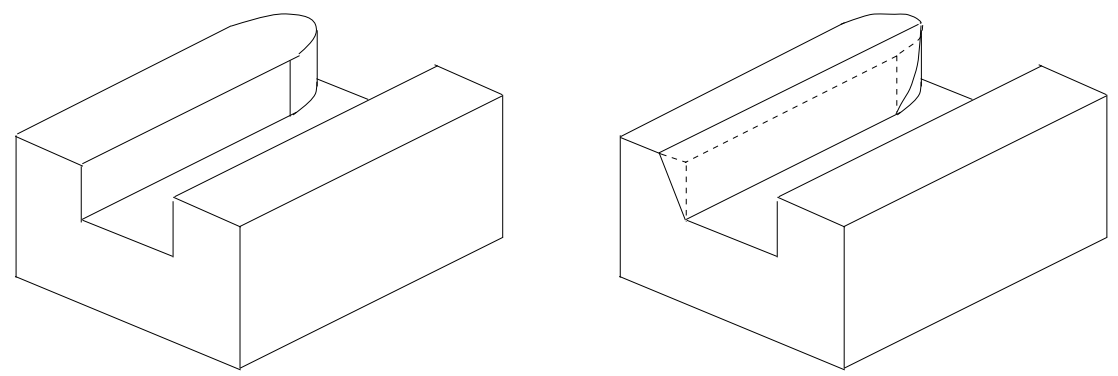

Figure 5: Left: Initial net shape. Right: Edited net shape.

If the exceptional changes concern explicit, user-defined constraints, then the user should be notified of the adjustment performed by the algorithm. This is a measure that accounts for the possibility that the ensuing shape change may violate a design choice that the editing client may have been unaware of.

An example is given in Figure 5. If the features were generated as shown in Figure 6, then constraint reconciliation is applicable. The designer's view, shown on the left of Figure 6, consists of two protrusions, labeled B and D, placed on top of block A. Protusion B is generated by extruding a rectangular cross section perpendicular to the top face of the block A. The cross section has width $d$ and height $h$. One of the ends of protrusion $\mathrm{B}$ has been rounded with a circular cut, C.

The view of the MPP client, shown on the right side of Figure 6, is different from that of the designer's. It consists of a block A from which a slot has been cut off. Since the angle $\alpha$ between edges $f_{1} \cdot e_{1}$ and $f_{1} \cdot e_{2}$ has a meaning to MPP, it has been explicitly established as an angular dimension. Similarly in the designer's view, the rounding of one of the slots sides has been generated with a circular cut, C.

Figure 7 shows how the MPP client would edit by giving a new value $\alpha^{\prime}$ to angle $\alpha$. The design client, to update its own view, first would compute a cross section to generate the new shape of extrusion $\mathrm{B}$ by replacing edges $b_{1} . e_{1}$
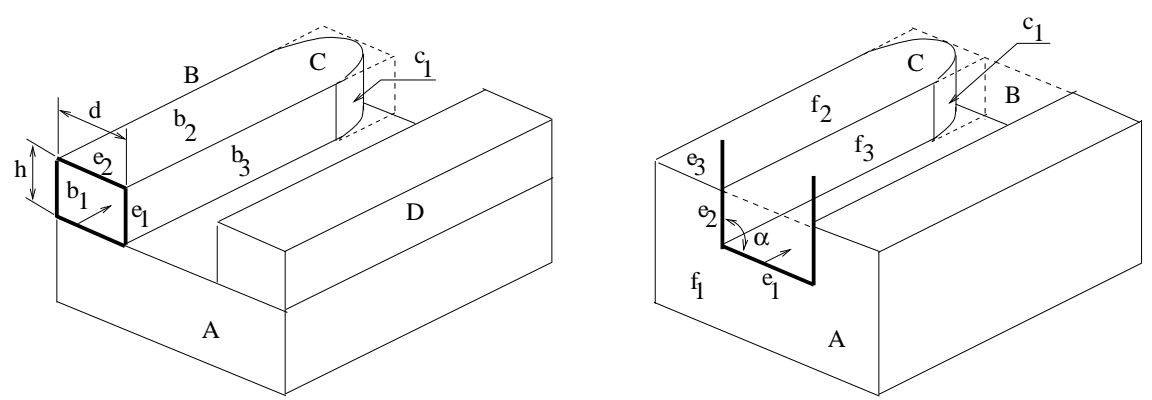

Figure 6: Design view left; MPP view right. Constraint reconciliation is possible. 

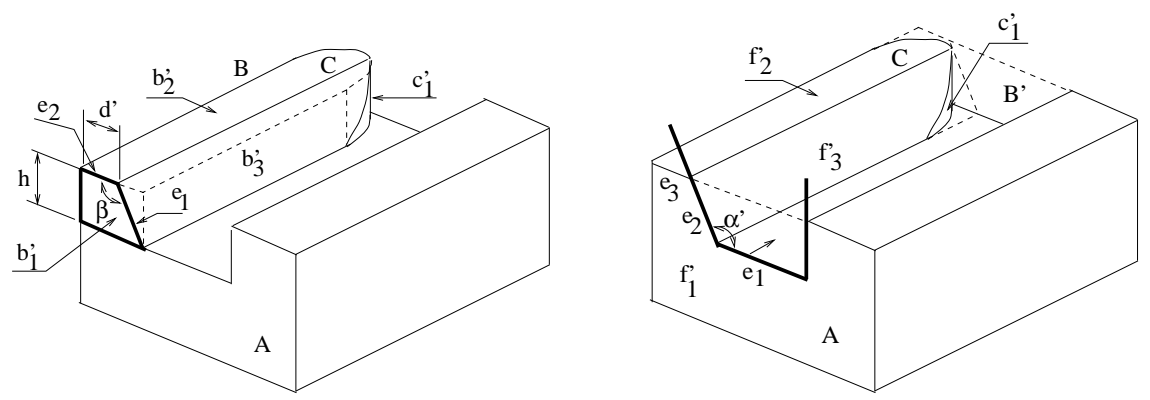

Figure 7: Left: Updated design view. Right: New MPP view.

and $b_{1} \cdot e_{2}$ with edges $f_{1}^{\prime} \cdot e_{2}$ and $f_{1}^{\prime} \cdot e_{3}$, respectively. Then, new values for the dimension constraints would be computed. Old dimension value $d$ would be now $d^{\prime}$ and the $90^{\circ}$ constant angle between edges $b_{1} \cdot e_{1}$ and $b_{1} \cdot e_{2}$ would be now a varying angle dimension with value $\beta$.

\subsection{History Adjustments}

Even though the constraint refers to prior geometry and elements of a single feature, it does not necessarily imply that we can adjust the cross section. Consider the design view shown in Figure 8. If the edit shown in Figure 7 has been carried out by the MPP client, the CAD client cannot update by constraint reconciliation alone. Here, the $\mathrm{CAD}$ client would have to use more general techniques. Let us show how a history-based approach can handle this situation.

The change client 1 performs is communicated to client 2 with the following information: The new net shape, and the change protocol that establishes a mapping between the shape elements of the old and the new net shapes. Client 2 examines the cross sections in an order that is compatible with its feature history. Keeping in mind that the net shape change is such that the number of features and their interdependence in the updating client remains the same, a feature is adjusted in the following situations:

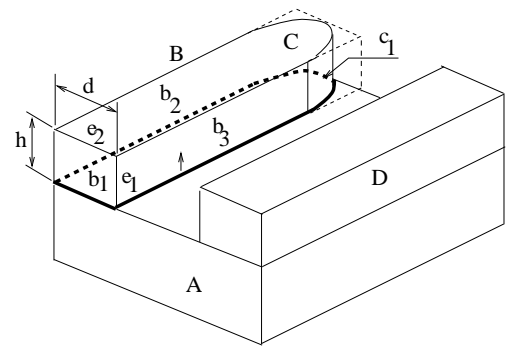

Figure 8: A different design view of the original net shape. Constraint reconciliation is not possible, and reinterpretation would be required. 
1. A cross section contributes to the old net shape a boundary element that has been changed in the new net shape;

2. The feature attachment method refers to a shape element that has been changed.

In each case, the cross section is recomputed for the new net shape and the attachment method validated. If the cross section cannot be adjusted consistent with the new feature shape, a question that is answered generatively, we attempt elementary reinterpretation. If that fails as well, then this attempt at feature adjustment fails. We can then backtrack and try a different feature sequence.

Note that feature attachment induces a partial order on features. If feature $A$ uses geometric elements of feature $B$, to define the cross section or the attachment method, then the creation of $B$ must precede the creation of $A$. The partial order will be respected by the adjustment algorithm, but need not determine a unique order. We allow the possibility of backtracking if, in the chosen

order, adjustment fails. For the next feature in a particular order, we obtain Algorithm 1

\section{Algorithm 1}

1. For the next feature contributing to those net shape elements that have changed, recompute the cross section of the feature from the intermediate, local geometry, and from the net shape.

2. Adjust the geometry of the cross section by filling in the missing elements and deleting the elements no longer in use. If the cross section cannot contribute to the new net shape, delete the feature.

3. Confirm that the feature generation method (extrusion or revolution) can be maintained. If not, attempt reinterpretation and report such changes, otherwise fail.

4. Recompute the geometric constraints: For a dimensional constraint, compute the new value if any. For a logical constraint, change incidence to distance if necessary, and change perpendicular or parallel to angle, if necessary. Delete tangency constraints if necessary. Report all changes other than changes to dimensional constraint values.

5. Recompute the attachment method. If the method has to be changed, for example going from through-next to blind or through-all, then report the change. If no suitable attachment method can be generated, fail.

We compute the cross section with Algorithm 1C. 


\section{Algorithm 1C}

1. Intersect the sketching plane with the prior geometry that client 2 has constructed so far and with the net shape. Intersections with the prior geometry are called prior elements, the others new elements.

2. If prior elements correspond to new elements, by the change protocol, replace the prior elements with new elements.

3. Fill in gaps between prior and replaced cross section elements with segments of new elements, if the segments come from surfaces compatible with the feature generation method.

4. Fill in remaining gaps between prior and replaced elements by extending line segments and arcs.

5. If gaps remain, close each with a line segment or fail.

Reinterpretation is attempted as follows, for revolved features:

\section{Algorithm 1R}

1. Identify all surfaces belonging to the revolved feature.

2. Group the surfaces into sets that are cylindrical with the same cross section, and surfaces that cannot be so generated.

3. For each cylindrical group, generate a cross section. If the feature component can be generated with the available attachment rules, do it. Otherwise fail.

4. If surfaces remain that have not yet been generated, fail.

Similarly, for extruded features, two separate algorithms are used, one for generating a different direction of sweeping, the other for generating a set of revolved features.

The main difficulty for the algorithm is the problem of gap filling. Sections from the new net shape are especially appropriate if they do not correspond to later features in the structure of client 2. Allowing only those new elements that come from compatible surfaces may be too conservative in view of feature reinterpretation, and a broader approach would combine attempts to reinterpret the generation method with gap filling.

We can think of cases where remaining gaps can be filled with suitable curves that do not contribute to the net shape. Finding such curves in general would be difficult. Verifying that a particular curve $C$ is appropriate, on the other hand, is easy: Use the curve and observe whether the final net shape generated by client 2 has a surface element that came from $C$. 

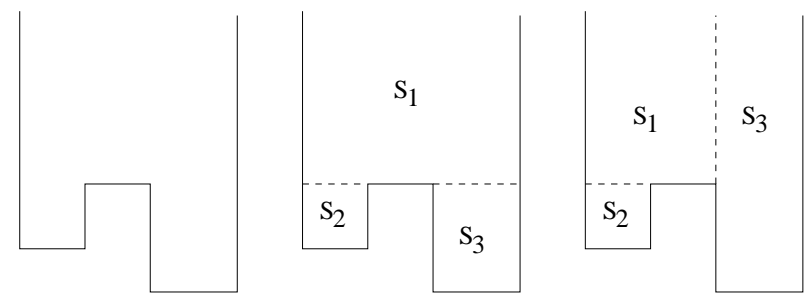

Figure 9: Compound cut. Left: Design view. Middle: MPP view. Right: A different MPP view.

\section{Feature Addition and Deletion}

Algorithm 1 assumes that the net shape change is such that the number of features, and their interdependence, in client 2, remains the same. This may not always be possible, especially if the editing operation consisted of the addition or deletion of a feature. We consider this situation now, assuming that a deleted feature, in the editing client, did not have other features depending on it. Such a feature would be a leaf feature in the dependency tree. If nonleaf features are to be deleted, then we would either delete the dependent features first, or request that the editing client restructure its design such that the dependencies are eliminated. The latter operation is familiar from commercial CAD systems.

There are two fundamental issues that arise when adding or deleting a feature. First, the feature vocabulary of the editing client may be richer than that of the updating client. For instance, the CAD client can add protrusions, but the MPP client may be restricted to cuts from a stock shape only. Second, the feature structure of the editing client may be substantially different from the structure of the updating client. For example, the CAD client may be adding a single profiled cut, but the MPP client may have to decompose the profile into a compound structure of simpler profiles.

\subsection{Adding a Protrusion or a Cut}

The single feature added is, by definition, a leaf feature. If the updating client has a compatible feature type, then the change requires constructing a new cross section and generating the appropriate feature. Difficulties arise for incompatible feature vocabularies.

When the CAD client adds a profiled cut, the difficulty for the MPP client may be that the cut must be decomposed into several cuts accounting for available machining processes. The problem is solved by tiling the CAD profile with elementary profiles that cover it. For instance, consider the cut profile of Figure 9. The profile on the left can be decomposed into three rectangular cuts, $S_{1}, S_{2}, S_{3}$. Note that different decompositions are possible. Tiling algorithms are easy to devise. More sophisticated tilings would consider cost, machining 


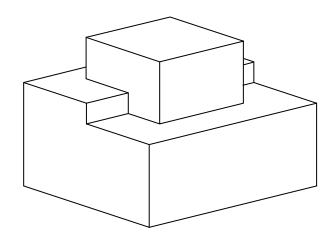

Figure 10: The CAD client adds the boss straddeling the step. An MPP client would have to update by enlarging the stock and adding several cut features.

characteristics, etc. See for example [17, 19].

A more difficult case is when the editing client has feature operations of a type not available to the updating client. For instance, assume that the CAD client adds a protrusion, but the MPP client can only add cut features to a base feature, the stock. Then the update for the MPP client is more difficult. An example is shown in Figure 10. Here, the adjustments of Algorithm 1 cannot succeed because the mapping between the old and the new net shapes does not identify the top face of the boss as belonging to the face set created by the base feature, i.e., the stock.

The example illustrates that for shape edits that are made with a richer feature vocabulary the updating client can succeed only if more general techniques from feature recognition are employed. Such techniques have been developed in, e.g., [5] and [14].

\subsection{Deleting a Protrusion or a Cut}

Since the deleted feature need not correspond to a single leaf feature in the updating client, several features may have to be adjusted using Algorithm 1. Moreover, the deletion of a protrusion, in the CAD client, may imply adding cut features in the MPP client. We also expect the possibility that features become redundant in the course of executing Algorithm 1, for instance when deleting a cut.

\section{Updating Attributes and Relations}

We consider changes that require updating secondary information associated with net shape elements. For example, consider the case where the CAD client does not maintain or process tolerancing information, material properties, surface finish, or engineering notes. We consider these information domains conceptually as relationships that are defined for net shape elements. In the case of unary relationships, such as surface finsih, it is customary to speak of at- 

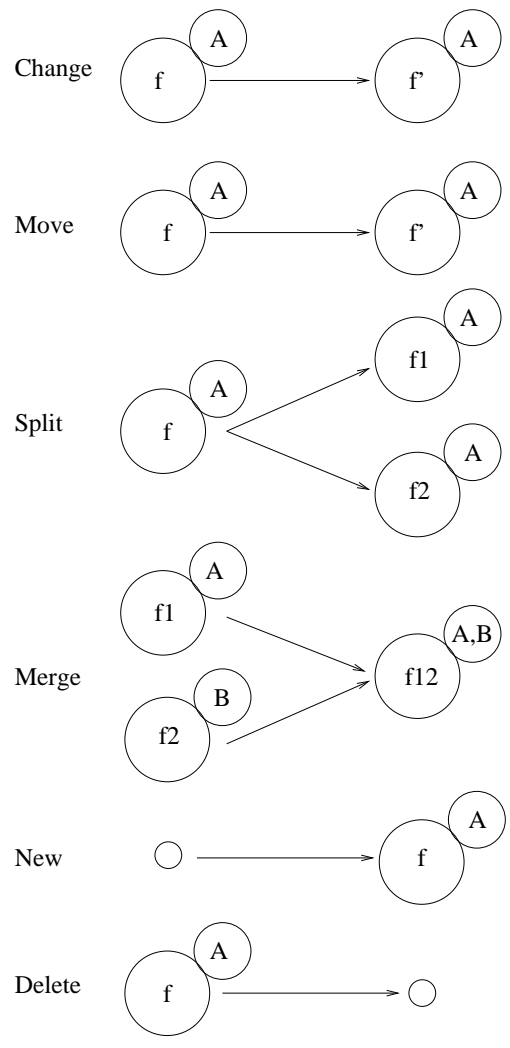

Figure 11: Graph description of changes undergone by net shape elements.

tributes. Whether the relationships are unary, binary or of higher degree, ${ }^{1}$ the basic problem is simply to maintain the associations correctly. This is done by transferring the associations, suitably edited, from the old to the new net shape.

As analyzed in [16], the mapping that relates the old net shape to the new net shape can be represented as a graph whose nodes are net shape elements and whose edges are operations on them. The operations are move, change, split, merge, delete, and new. The graph describes the changes each shape element of the old net shape undergoes and the net shape elements, if any, of the new net shape that correspond or to which a contribution is made. Figure 11 illustrates these concepts. Circles with lower-case letters represent net shape geometric elements. Circles with capital letters represent attributes associated with net shape elements. Because of the graph is directed and acyclic, updating associations is a good candidate for a rule-driven algorithm. The rules include computations on the net shape elements involved.

The design of the rules depends on the information domain. In [16], we

\footnotetext{
${ }^{1}$ We could represent form features as $n$-ary relationships on a suitable set of faces, edges and vertices.
} 
illustrated this approach using notes attached to net shape elements. Here, we consider a GD\&T attribute specifying a nominal dimension between two parallel faces with a parallel tolerance. The rules are applied at each edge at which there is an alteration of either face of the net shape. The rules are applied by "evaluating" the change protocol graph in the order of the changes, from the graph roots (elements of the old net shape) to the leaves (elements of the new net shape).

change: The net shape element has changed in area, but not in position and orientation. No action is required.

move: The net shape element has changed in position and orientation. If not in an intermediate position, evaluate the nominal dimension from the new position and orientation. If no longer parallel within tolerance, or if no longer within the nominal dimension range, notify user.

split: Replicate the GD\&T attribute and attach to all split descendants.

merge: Merge all attributes of the merged entities.

new: No action required.

delete: Notify user that the GD\&T attribute has been orphaned.

We see that the update activity for attributes is well-suited to a rule-based approach. The key operational devices needed in general are

1. Call a user-defined evaluation routine with a Boolean result.

2. Issue a user-defined notification. Notifications are minimally: attribute orphaned, attribute violated, attribute replicated.

3. Replicate an attribute.

4. Merge an attribute set.

Moreover, those devices are general, independent of the attribute domain, except for a call-back mechanism which is domain-dependent.

\section{Summary and Conclusions}

We have explored the requirements for the distributed maintenance of consistent master model information in a federated architecture, in which different software clients connect to a server and collaborate by disclosing information required by other clients to construct and maintain consistent views of the design. In particular, we have focused on maintaining shape-related information without forcing the CAD client to disclose proprietary design and editing information. 
In conjunction with earlier work, we believe it is possible to construct such a system that succeeds in automating a wide range of view updating operations.

We find that maintaining different feature views is complicated by the current, history-based CAD design style. In our algorithm to partially automate updates, we apply a core set of techniques familiar from the feature recognition literature when dealing with updating the feature history. In many situations an adjustment is possible purely by constraint reconciliation, a concept we introduced earlier. Were it not for the sequential design history implemented by CAD systems, constraint reconciliation would be much wider applicable. Finally, the maintenance of attributes can be completely automated, and, with it, the maintenance of many downstream views that can be derived from attributes and relations maintained on the net shape elements.

The limitations of our algorithms are less consequential in practice than might seem at first glance. We note that major changes of the net shape, such as the ones indicated in some of the examples, probably require human review by different individuals, so that automating radical design changes is not appropriate. Only routine changes should be automated. In view of this situation, it may not be worthwhile looking for perfect algorithms, from an applications point of view. Of course, a perfect history adjustment algorithm that can handle all possible situations would be an interesting technical accomplishment.

\section{References}

[1] IEEE Computer. Special issue on Concurrent Engineering, 26(1), 1993.

[2] Computer-Aided Design. Special issue on Network-Centric CAD, 30(6), 1998.

[3] V. Allada and S. Anand. Feautre-based modelling approaches for integrated manufacturing: state-of-the-art survey and future research directions. International Journal for Computer Integrated Manufacturing, 8(6):411-440, 1995.

[4] W.F. Bronsvoort and F.W. Jansen. Multi-view feature modelling for design and assembly. In J.J. Shah, M. Mäntylä, and D.S. Nau, editors, Advances in Feature Based Manufacturing, Manufacturing Research and Technology, 20, chapter 14, pages 315-330. Elsevier Science B.V., 1994.

[5] M.A. Chamberlain, A. Joneja, and T.C. Chang. Protrusion-features handling in design and manufacturing planning. Computer Aided Design, 25(1):19-28, 1993.

[6] X. Chen and C.M. Hoffmann. Towards feature attachment. Computer Aided Design, 27(9):695-702, 1995. 
[7] J.J. Cunningham and J.R. Dixon. Designing with features. The origin of features. In V.A. Tipnis and E.M. Patton, editors, Computers in Engineering Conference and Exhibition, volume 1, pages 237-243, San Francisco, 1988. ASME.

[8] K.J. de Kraker, M. Dohem, and W.F. Bronsvoort. Multiple-way feature conversion. opening a view. In M. Pratt, R.D. Siriram, and M..J. Wozny, editors, Product Modeling for Computer Integrated Design and Manufacture, pages 203-212, London, UK, 1997. Chapman and Hall.

[9] K.J. de Kraker, M. Dohmen, and W.F. Bronsvoort. Feature validation and conversion. In D. Roller and P. Brunet, editors, CAD Systems Development. Springer Verlag, Heidelberg, 1997.

[10] K.J. de Kraker, M. Dohmen, and W.F. Bronsvoort. Multiple-way feature conversion to support concurrent engineering. In M. Pratt, R.D. Siriram, and M.J. Wozny, editors, Product Modeling for Computer Integrated Design and Manufacture, pages 203-212, London, UK, 1997. Chapman and Hall.

[11] T. DeMartino, B. Falcidieno, and S. Hassinger. Design and engineering process integration through a multiple view intermediate modeller in a distributed object-oriented system environment. Computer-Aided Design, 30(6):437-452, May 1998.

[12] M. Dohmen, K.J. de Kraker, and W.F. Bronsvoort. Feature validation in a multiple-view modeling system. In 16th ASME International Computers in Engineering Conference, Irvin, NY, USA, 19-22 August 1996. ASME.

[13] J. Han and A.A.G. Requicha. Incremental recognition of machining features. In Proceedings of the ASME Computers in Engineering Conference, pages 587-598, Minneapolis, MN, 1994.

[14] J.H. Han and A.A.G. Requicha. Integration of feature based design and feature recognition. Computer-Aided Design, 29(5):393-403, May 1997.

[15] J.H. Han and A.A.G. Requicha. Modeler-independet feature recognition in a distributed environment. Computer-Aided Design, 30(6):453-463, May 1998 .

[16] C.M. Hoffmann and R. Joan-Arinyo. CAD and the product master model. Computer-Aided Design, 1998. To appear.

[17] A. Kusiak. Optimal selection of machinable volumes. Transactions of Institute of Industrial Engineers, 22(2):151-160, 1990.

[18] T. Laakko and M. Mäntylä. Feature modelling by incremental feature recognition. Computer-Aided Design, 25(8):479-492, August 1993. 
[19] D.S. Nau, G. Zhang, and S.K. Gupta. Generation and evaluation of alternative operation sequences. In A.R. Thangaraj, A. Bagchi, M. Ajanappa, and D.K. Anand, editors, Quality Assurance Through Integration of Manufacturing Processes and Systems. ASME, 1992. (Proc. 1992 ASME Winter Annual Meeting, Publication No. PED-Vol. 56, pp. 93 - 108).

[20] H. Suh and R.S. Ahluwalia. Feature generation in concurrent engineering environment. In J. Rossignac and J. Turner, editors, Symposium on Solid Modelling Foundations and CAD/CAM Applications, pages 493-502, Austin, TX, June 5-7 1991. ACM Press.

[21] Y.-J. Tseng and S.B. Joshi. Recognizing multiple interpretations of interacting machining features. Computer-Aided Design, 26(9):667-688, 1994.

[22] X. Xu and S. Hinduja. Recognition of rough machining features in $21 / 2$ D components. Computer-Aided Design, 30(7):503-516, 1998. 